\title{
Minimum cover set coverage algorithm for achieving low energy in wireless sensor networks
}

\author{
AIMIN WANG ${ }^{1,2}$, YINGNAN GAO ${ }^{1}$, WENJUAN JIA ${ }^{3, *}$ \\ 1 College of Computer Science and Technology, Jilin University, Changchun, Jilin \\ 130012, China \\ 2 Key Laboratory of Symbolic Computation and Knowledge Engineering of Ministry \\ of Education, Jilin University, Changchun, Jilin 130012, China \\ 3 College of software, Jilin University, Changchun, Jilin 130012, China
}

\begin{abstract}
A minimum cover set coverage algorithm (MCSCA) for pursuing low energy is presented in this paper to prolong the lifetime of wireless sensor networks. The proposed algorithm improves energy efficiency in three aspects. First, when generating cover sets, the selection strategy of the algorithm considers the contributions of sensor nodes, energy variance, and other factors. The algorithm can cover all targets with a few sensor nodes. Second, useless coverage optimization reduces coverage areas without target nodes to save energy. Third, redundant coverage optimization further saves energy by reducing redundant coverage in wireless sensor networks. Compared with similar heuristic algorithms, the proposed MCSCA can extend network lifetime by $11 \%$ on average.
\end{abstract}

\section{Introduction}

Coverage is a critical problem in wireless sensor networks. According to the monitored objects and requirements, the coverage problem can be divided into the following categories [1]: area coverage, target coverage, and barrier coverage. Sleep scheduling is very beneficial in prolonging network lifetime.

In the present work, we propose a minimum cover set coverage algorithm (MCSCA) for pursuing low energy in wireless sensor networks. The proposed algorithm comprises three phases: cover set generation, useless coverage optimization, and redundant coverage optimization. The MCSCA can produce both non-disjointed cover sets and disjointed cover sets. Through a sleep scheduling mechanism, the cover sets produced by the MCSCA can work alternately with minimum energy consumption to maximize network lifetime.

\section{Related Works}

Chuan-Kang Ting and Chien-Chih Liao [2] proposed the memetic algorithm (MA) to solve coverage problems. The MA combines the theory of Darwinian evolution mechanism and local promotions. This algorithm uses global and local searches to find the approximate optimal solution. Sensors are encoded as chromosomes that present all cover sets. Crossover and mutation are implemented on chromosomes to produce an approximate optimal coverage scheme. The MA can effectively deal with NP complete problems. Unlike the integer-coded genetic algorithm (IGA) [3], the MA does not need to calculate the upper boundary of the number of cover sets. The improved MA (IMA) [4] improves the MA by using matrixes to present chromosomes. A matrix can intuitively show the coverage relationship between sensor nodes and target nodes. Different local promotion strategies are used in the IMA. Thus, the IMA can more easily produce approximate optimal solutions than the MA. However, the IGA, MA, and IMA can only produce disjointed cover sets and do not utilize the advantage of non-disjointed cover sets in prolonging network lifetime.

The MCSCA has the following advantages over the target coverage algorithms mentioned previously. (1) It can produce both disjointed and non-disjointed cover sets. (2) It can complete coverage tasks with a few sensors. (3) It can reduce useless coverage and redundant coverage as much as possible in networks. 


\section{Assumptions and definitions}

The monitoring area is assumed to be a 2D space. A certain number of interest points exist in the target area. Sensor nodes and target nodes are uniformly and randomly deployed in the area. To simplify the energy consumption analysis, we only consider the energy consumption of the sensing function and disregard the energy consumption of transmission and calculation.

Definition 1 (target nodes and sensor nodes)

Target set: $T=\left\{t_{1}\left(x_{1}, y_{1}\right), t_{2}\left(x_{2}, y_{2}\right), \ldots . . t_{m}\left(x_{m}, y_{m}\right)\right\}$

Sensor set: $S=\left\{s_{1}\left(x_{1}, y_{1}\right), s_{2}\left(x_{2}, y_{2}\right), \ldots . . . s_{n}\left(x_{n}, y_{n}\right)\right\}$

Definition 2 (probabilistic sensing model)

The probability of target ti is covered by sensor si.

$$
\begin{aligned}
p\left(s_{i}, t_{j}\right) & = \begin{cases}0 & r_{i}<\operatorname{dst}\left(s_{i}, t_{j}\right) \\
1 & r_{i} \geq \operatorname{dst}\left(s_{i}, t_{j}\right)\end{cases} \\
\operatorname{dst}\left(s_{i}, t_{j}\right) & =\sqrt{\left(x_{i}-x_{j}\right)^{2}+\left(y_{i}-y_{j}\right)^{2}}
\end{aligned}
$$

Definition 3 (energy consumption model)

We used an energy consumption model similar to that in [18].

$E_{i}\left(r_{s}\right)=\boldsymbol{\theta} \cdot r_{s}^{2}$

\section{Proposed Algorithm}

\subsection{Algorithm Structure}

On the basis the algorithm presented in [5], we propose the centralized coverage algorithm MCSCA, which has two phases of optimization. The algorithm can produce both disjointed cover sets and non-disjointed cover sets. It consists of three parts: (1) generation of cover set using a selection strategy for choosing reasonable sensor nodes, (2) useless coverage optimization to reduce coverage areas without target nodes, and (3) redundant coverage optimization to reduce multiple target node coverage.

The MCSCA produces one cover set per round. The sensor nodes in the cover set may work with different radiuses. When all the sensor nodes run out of energy or when the remaining sensors in the network cannot cover all the targets, the MCSCA ends.

The MCSCA first enters the phase in which a cover set is generated. Proper sensor nodes are added to the cover set through a selection strategy. Following the generation of the cover set, two phases of optimization are implemented on the cover set. Useless coverage optimization aims to reduce the coverage area without target nodes. In the case of guaranteeing complete coverage, optimization can reduce energy waste. Redundant coverage optimization is carried out on the result of the previous optimization phase. This second phase of optimization saves energy further by preventing a target from being covered by multiple sensor nodes. The MCSCA constantly repeats the above three phases to schedule sensor nodes. When the termination conditions are met, the MCSCA ends.

\subsection{Selection of appropriate sensor nodes}

The targets covered by a sensor node can be covered or uncovered targets. At one point, the fitness of a sensor node is influenced by target nodes that are covered by such node and the other $\mathrm{X}$ covered by other nodes.

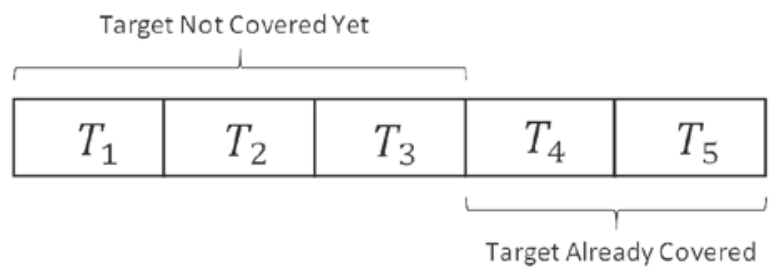

Figure 2. Target nodes covered by a sensor node

Figure 2 shows that target nodes T1-T5 are covered by a sensor node. A large proportion of uncovered target nodes corresponds to a long network lifetime. This principle helps minimize the use of sensor nodes and reduce energy waste from redundant coverage.

The phenomenon in which a new cover set cannot be generated because a few targets cannot be 
covered is called the "single-point bottleneck." This bottleneck can significantly shorten network lifetime. It can be avoided under a balanced network energy, which can be achieved with a cover set whose energy variance is small.

The sum of the energy of sensor nodes as they cover a common target node is called the "coverage energy" of targets. The target with minimum coverage energy is called "critical target."

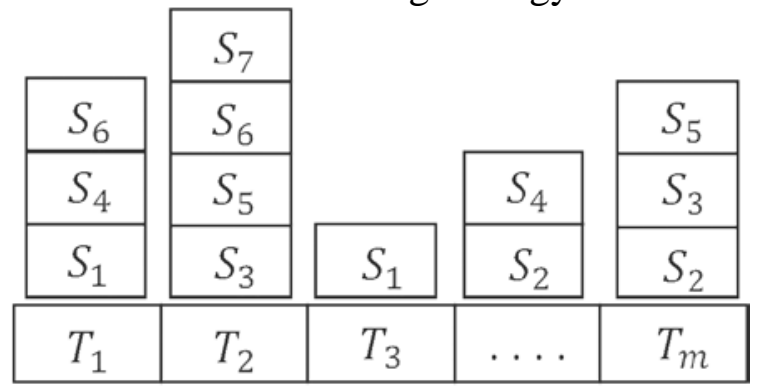

Figure 3. Network coverage

In Figure 3, T3 is a critical target node that influences the generation of cover sets. The selection strategy should avoid selecting several sensor nodes that can cover all critical targets per round. This principle can balance the energy consumption of the network.

\subsection{Algorithm description}

Based on the algorithm presented in [5], the MCSCA uses an appropriate selection strategy and implements two phases of optimization.

Fitness measures the coverage contribution of a sensor. When some targets cannot be covered by any sensor node, the algorithm ends. The selection strategy evaluates sensor node fitness from four aspects:

fitness $=\theta *$ Coverage_Rate $+\varphi *$ Energy_Rate $+\gamma *$ Variance_Rate $+\varepsilon *$ RemainingEnergy_Rate

(1) Coverage_Rate

Coverage_Rate describes the relationship between uncovered target nodes and covered target nodes.

Coverage_Rate $=1-$ (redundancy/all_covered)uncovered

Redundancy/all_covered represents the proportion of covered target nodes. The exponent "uncovered" indicates that the fitness of a sensor node is greatly affected by the number of uncovered target nodes. The "Coverage_Rate" formula can appropriately evaluate the sensor node from the aspect of coverage condition. In different circumstances, we can change the values of $\theta, \varphi$, and $\gamma$ to adjust the emphasis of the selection strategy.

(2) Energy_Rate

$$
\begin{aligned}
\text { Energy_Rate } & =E R(\text { redundancy }) \\
& \left.=\left(\sum_{i=1}^{k}\left(s / \text { energy_sum }\left(N S\left(t_{i}\right)\right)\right)\right) / k\right) \quad t_{i} \in \text { redundancy, } k=\mid \text { redundancy } \mid
\end{aligned}
$$

the sensor node with a small value of Energy_Rate is preferable. Choosing the sensor node with a large Energy_Rate easily causes a single-point bottleneck.

(3) Variance_Rate

If the energy consumptions of sensor nodes are not balanced, single-point bottlenecks are likely to occur. This phenomenon tends to shorten network lifetime. When the MCSCA generates a cover set, the energy variance of sensor nodes must be considered.

(4) RemainingEnergy_Rate

$$
\text { Variance _ Rate }=\operatorname{VR}\left(C_{k} \cup s\right)
$$

\section{RemainingEnergy_Rate $=$ senergy/Initial_Energy}

Sensor nodes with high energy should be preferable, and narrowing the gap of sensor node energy is an effective way to balance energy consumption.

Useless coverage optimization can reduce useless coverage in the network. After generating a cover set, the radiuses of all sensor nodes are Rmax. Useless coverage may occur in this phase of optimization. In the case of guaranteeing original coverage, the radiuses of sensor nodes are shortened to save energy. 


$$
\text { Energy_Saving }=\partial * \sum_{f=1}^{t}\left(R_{\max }^{2}-r_{f}^{2}\right) \quad t=\left|C_{k}\right|
$$

In the formula, "Energy_Saving” represents the amount of energy that is saved by reducing useless coverage. rf is the radius after adjustment.

Redundant coverage optimization is carried out after useless coverage optimization. energy can be saved further by reducing redundant coverage in the network. The weights of sensor nodes are calculated according to radius variation and the remaining energy. The sensor node with maximum weight maintains its radius because maximum weight indicates that the sensor node has high energy and energy can be saved by reducing the radius. The radiuses of the remaining sensor nodes are shortened.

$$
\begin{aligned}
\text { weight } & =\text { weight } \_ \text {function }\left(s_{i}\right) \\
& =\alpha *(1-(\text { diff / original_radius }))+\beta *\left(s_{i} \text {.energy / Initial_Energy }\right)
\end{aligned}
$$

In the formula, “original_radius" is the radius of the sensor node after useless coverage optimization. "Diff" represents the radius variation in redundant coverage optimization. Little energy is saved when the value of "diff/original_radius" is small, and a high remaining energy equates to a long network lifetime. Hence, the radius of the sensor node with high energy and a small "diff" must remain unchanged. $\alpha$ and $\beta$ can be changed to meet the requirements.

\section{Simulation and Analysis}

We use MATLAB to examine the performance of the MCSCA. Targets and sensor nodes are randomly and uniformly deployed into a $100 \mathrm{~m} \times 100 \mathrm{~m}$ area. The number of targets is 40, For each simulation, we generate 30 topologies and execute each algorithm 30 times on each topology. The algorithm is compared with $\mathrm{B}\{\mathrm{GOP}\}$, static-CCF, and dynamic-CCF. All the algorithms are utilized under the same condition. the number of sensor nodes ranges from 200 to 400 .

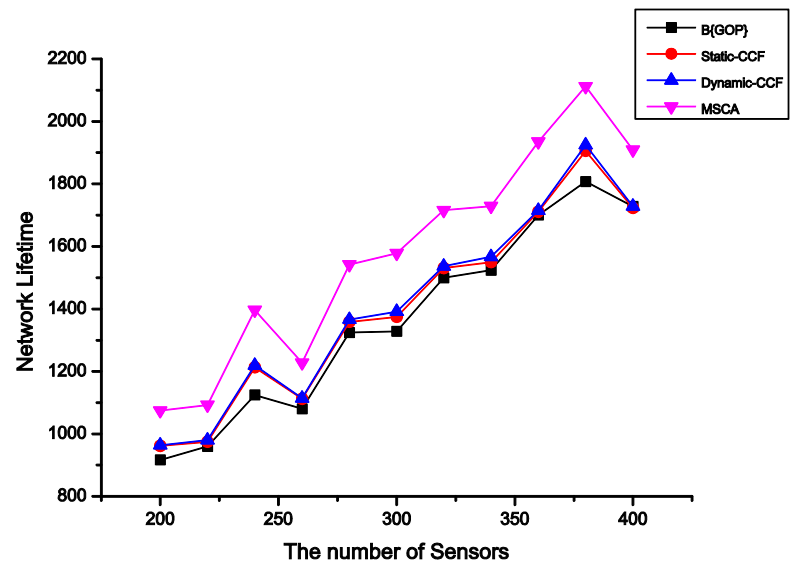

Figure 4. Influence of the number of sensor nodes on network lifetime

Figure 4 shows the influence of the number of sensor nodes on network lifetime. For all the four algorithms, network lifetime grows with an overall increase in the number of sensor nodes. Compared with $\mathrm{B}\{\mathrm{GOP}\}$, static-CCF, and dynamic-CCF, the MCSCA prolongs the network lifetime by $11 \%$ on average. When the number of sensor nodes is 240 , the extension of network lifetime increases to $14 \%$. 


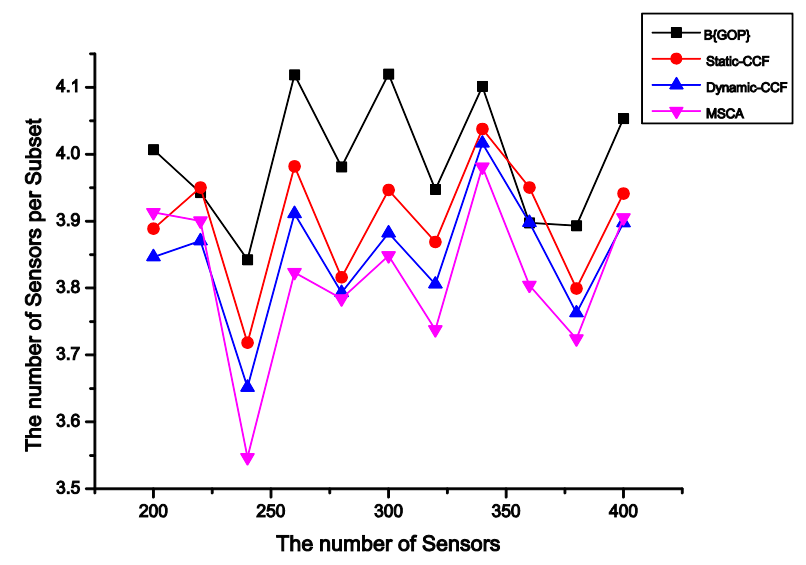

Figure 5. Influence on the average number of sensor nodes per cover set

In figure 5, the MCSCA uses fewer sensor nodes than the other three algorithms when generating a cover set. Its selection strategy can reasonably use a few sensor nodes to cover all targets during cover set generation.

\section{Conclusion}

To solve the energy efficiency coverage problem in wireless sensor networks, we propose a novel heuristic algorithm that can improve the energy efficiency of wireless sensor networks and consequently prolong their lifetime. In this algorithm, energy efficiency is improved from three aspects. First, appropriate sensor nodes are added to the cover set by a selection strategy. Second, useless coverage optimization reduces the coverage area without targets to save energy. Third, redundant coverage optimization is conducted to further save energy by reducing redundant coverage. Above all, the MCSCA can generate cover sets with few sensor nodes and consume little energy to prolong network lifetime. In the case of covering all targets in the area, the MCSCA performs better than $\mathrm{B}\{\mathrm{GOP}\}$, static-CCF, and dynamic-CCF, particularly in terms of prolonging network lifetime.

\section{REFERENCES}

[1] Ali Ahmadi, Mohammad Shojafar, SeyedeFatemeh Hajeforosh, Mehdi Dehghan, and MukeshSinghal. An efficient routing algorithm to preserve k-coverage in wireless sensor networks. The Journal of Supercomputing, 68(2):599-623, 2014.

[2] Chuan-Kang Ting and Chien-Chih Liao. A memetic algorithm for extending wirelesssensor network lifetime. Information Sciences, 180(24):4818-4833, 2010.

[3] Chih Chung Lai, Chuan Kang Ting, and Ren Song Ko. An effective genetic algorithmto improve wireless sensor network lifetime for large-scale surveillance applications. InEvolutionary Computation, 2007. CEC 2007. IEEE Congress on, pages 3531-3538.

[4] D. Arivudainambi, S. Balaji, and D. Rekha. Improved memetic algorithm for energyefficient target coverage in wireless sensor networks. In Networking, Sensing and Control(ICNSC), 2014 IEEE 11th International Conference on, pages 261-266.

[5] Dimitrios Zorbas, Dimitris Glynos, Panayiotis Kotzanikolaou, and Christos Douligeris.Solving coverage problems in wireless sensor networks using cover sets. Ad Hoc Networks,8(4):400-415, 2010. 